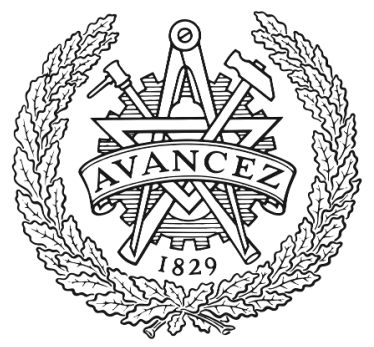

CHALMERS

UNIVERSITY OF TECHNOLOGY

\title{
Ab initio investigation of monoclinic phase stability and martensitic transformation in crystalline polyethylene
}

Downloaded from: https://research.chalmers.se, 2023-04-26 11:37 UTC

Citation for the original published paper (version of record):

Olsson, P., Hyldgaard, P., Schröder, E. et al (2018). Ab initio investigation of monoclinic phase stability and martensitic transformation in crystalline polyethylene. Physical Review Materials, 2(7): 7-13.

http://dx.doi.org/10.1103/PhysRevMaterials.2.075602

N.B. When citing this work, cite the original published paper. 


\title{
$A b$ initio investigation of monoclinic phase stability and martensitic transformation in crystalline polyethylene
}

\author{
Pär A. T. Olsson, ${ }^{1,2, *}$ Per Hyldgaard, ${ }^{3}$ Elsebeth Schröder, ${ }^{3}$ Elin Persson Jutemar, ${ }^{4}$ Eskil Andreasson, ${ }^{4,5}$ and Martin Kroon ${ }^{6}$ \\ ${ }^{1}$ Materials Science and Applied Mathematics, Malmö University, SE-205 06 Malmö, Sweden \\ ${ }^{2}$ Division of Mechanics, Lund University, Box 118, SE-22100 Lund, Sweden \\ ${ }^{3}$ Department of Microtechnology and Nanoscience (MC2), Chalmers University of Technology, SE-412 96 Gothenburg, Sweden \\ ${ }^{4}$ Tetra Pak, Ruben Rausings gata, SE-221 86 Lund, Sweden \\ ${ }^{5}$ Department of Mechanical Engineering, Blekinge Institute of Technology, SE-371 79, Karlskrona, Sweden \\ ${ }^{6}$ Department of Mechanical Engineering, Linnaeus University, SE-351 06 Växjö, Sweden
}

(Received 25 April 2018; published 10 July 2018)

\begin{abstract}
We study the phase stability and martensitic transformation of orthorhombic and monoclinic polyethylene by means of density functional theory using the nonempirical consistent-exchange vdW-DF-cx functional [Phys. Rev. B 89, 035412 (2014)]. The results show that the orthorhombic phase is the most stable of the two. Owing to the occurrence of soft librational phonon modes, the monoclinic phase is predicted not to be stable at zero pressure and temperature, but becomes stable when subjected to compressive transverse deformations that pin the chains and prevent them from wiggling freely. This theoretical characterization, or prediction, is consistent with the fact that the monoclinic phase is only observed experimentally when the material is subjected to mechanical loading. Also, the estimated threshold energy for the combination of lattice deformation associated with the T1 and $\mathrm{T} 2$ transformation paths (between the orthorhombic and monoclinic phases) and chain shuffling is found to be sufficiently low for thermally activated back transformations to occur. Thus, our prediction is that the crystalline part can transform back from the monoclinic to the orthorhombic phase upon unloading and/or annealing, which is consistent with experimental observations. Finally, we observe how a combination of such phase transformations can lead to a fold-plane reorientation from $\{110\}$ to $\{100\}$ type in a single orthorhombic crystal.
\end{abstract}

DOI: 10.1103/PhysRevMaterials.2.075602

\section{INTRODUCTION}

The mechanical properties of semicrystalline polymers are largely dictated by the microstructure and degree of crystallinity of the polymer, which can vary substantially depending on the manufacturing technique and process settings. This is attributed to the fact that the mechanical response of the crystalline and amorphous regions are widely different, and their respective parts interplay and jointly contribute to the macroscopic properties. In terms of yielding, typically the amorphous parts are ductile and can accommodate substantial amounts of strain before rupture, and the crystalline parts behave in a more brittle manner with the plastic deformations constrained by preferential crystallographic orientations [1-4]. Thus, by varying the content of such phases and the process strategy, widely different mechanical properties of semicrystalline polymers can be obtained [1,5], which complicates the prediction of the mechanical response. In light of the large number of industrial applications for such materials, ranging from lightweight components in the automotive industry to sealing screw caps for beverage and food packages, this issue inhibits the capability to optimize the product performance and to minimize the material expenditure.

The microstructure of high-density linear polyethylene (PE) typically consists of a spherulitic semicrystalline arrangement with alternating crystalline lamellae and amorphous layers [6].

*par.olsson@mau.se
Under normal conditions the lamellae structures are commonly lozenge shaped crystals with the polymer chains ordered according to an orthorhombic unit cell, arranged such that the crystal side surfaces are terminated by $\{110\}$ faces, while the lamella surface normal direction coincides with the [001] direction [6]. The polymer chains are folded back and forth into the lamellae multiple times, such that they are primarily aligned parallel to the orthorhombic $c$ axis. This implies that bonding in the $c$ direction is dominated by strong covalent bonding, whereas weak van der Waals (vdW) interaction is the predominant bonding type in the transverse directions. The lamellae are interconnected via tie molecules that extend through the amorphous region to the neighboring lamella and possibly even further. Such tie molecules and entangled polymer segments are responsible for holding the polymer together. The density of the polymer and the molecular structure depend on the manufacturing technique and process timings, but the size of the crystalline lamellae thickness typically lies in the range 3-25 $\mathrm{nm}$, whereas the lateral dimensions can reach up to $50 \mu \mathrm{m}[6-9]$.

Predicting the deformations and mechanical response of semicrystalline polymers is a nontrivial task, since it involves the interplay of mechanisms at different length scales [2]. Yielding of amorphous polymers is characterized by slippage of the random chains, and the response depends strongly on the chain entanglements. On the other hand, for crystalline PE three important crystal plastic mechanisms have been identified: (i) slip [8-21], (ii) twinning [8,13,22-24], and (iii) martensitic transformation $[8,16,22,23,25-31]$. 
The occurrence of martensitic transformation in polymer crystals was initially indicated by the observation of extra reflections in $\mathrm{x}$-ray and electron diffraction patterns when studying deformed crystals [22,26-29,32]. It was found that the orthorhombic crystal transforms into that of a monoclinic lattice [26]. Because such transformations typically cannot accommodate the same amount of strain as slip, the deformations are primarily found to accompany the early onset of yielding for situations when slip is geometrically unfavorable. Thus, to facilitate the slip, the crystal reorients to restore the geometrical orientation such that glide can be initiated [9]. Owing to the chain orientation and the covalent nature in the chain direction, martensitic transformations of crystalline PE occur exclusively by transverse deformations in the plane perpendicular to the chain direction. The most plausible modes and those observed experimentally belong to the $\mathrm{T} 1$ and $\mathrm{T} 2$ families of transformation $[16,22,23,26]$ (referred to as A and B, respectively, in [26]), which require the least amount of shearing strain. Despite their frequent experimental observation, knowledge of the transition strain energy and chain shuffling activation energy remains unknown, which partly motivates this study.

Beside these issues, the mechanical stability of the monoclinic phase is a subject of debate. Experiments have indicated that the monoclinic structure may be metastable or unstable and is present only when it is subjected to stress. This conjecture is based on the observation that deformed crystals transform back to the orthorhombic structure upon relaxation and/or annealing [33-35]. Based on x-ray diffraction experiments, the mechanism by which the monoclinic phase transforms back to the orthorhombic phase upon annealing has been investigated [34]. It has been found to concur with that of the propagation of the orthorhombic(110)/monoclinic(010) interface towards the monoclinic side of the crystal, in accordance with the predictions of the $\mathrm{T} 1$ and $\mathrm{T} 2$ transformation mechanisms. Moreover, the fold planes of lamellae have been found to change from $\{110\}$ to $\{100\}$ post-annealing. Such behavior could be an implication that the monoclinic structure is merely an intermediate configuration to accommodate foldplane transformations $[7,8,36]$. Hence, the stability of the free-standing monoclinic phase remains elusive, which merits further investigation.

In the present work we address these issues through a theoretical atomistic exploration of the orthorhombic-tomonoclinic phase transition and a discussion of its importance for understanding mechanically induced phase transitions in the PE system. Primarily, we seek to answer three questions with our computational analysis: (i) How are the preferential martensitic transition paths between the orthorhombic and monoclinic phases characterized in terms of threshold energy, chain shuffling, as well as strain energy and volume conservation? (ii) Under what conditions is the monoclinic phase stable? (iii) What is the nature of the stabilization of the orthorhombic phase at zero pressure? For the first issue, we compute the strain energy landscape associated with the martensitic transformation and the activation energy connected with chain shuffling. Such knowledge and data provide valuable discrete dislocation and crystal plasticity input for macroscopic modeling. For the second, we study the phonon characteristics with the intention to characterize whether the monoclinic phase is stable or not. For the third question, we correlate the phase differences in cohesive energies with the changes emerging in the electron density variation and with the variation in the interchain $\mathrm{vdW}$ bonding.

Our analysis is based on first-principle density functional theory (DFT) using the vdW density functional (vdW-DF) method [37-39] in the recent consistent-exchange formulation vdW-DF-cx $[39,40]$. This nonlocal correlation functional is based on rigorous physical insight and can be expected to be transferable with the capability to provide robust predictions of materials behavior, free of all experimental input. The vdW-DF-cx applicability is documented for the PE system and it allows us to detail the nature of the molecular movement that is associated with the martensitic phase transformation [21].

The remainder of the paper is organized as follows. In Sec. II we introduce the simulation setups and provide computational details. Results are presented and discussed in Secs. III and IV, respectively, while Sec. V contains our summary and conclusions.

\section{SIMULATION SETUP AND COMPUTATIONAL DETAILS}

\section{A. Investigated phases}

The two phases considered in the present work correspond to the orthorhombic (Pnam space group) and monoclinic $(C 2 / m$ space group) phases, see Figs. 1(a) and 1(b), respectively. X-ray and neutron diffraction measurements at ambient temperature (4-90 K) have revealed that the experimental orthorhombic lattice parameters correspond to $a=7.12-7.42 \AA$, $b=4.85-4.96 \AA$, and $c=2.54-2.55 \AA$ [42-45], where the latter dimension is aligned with the polymer chain direction. As depicted in Fig. 1(a), the molecular units are arranged such that the chains are rotated by about $45^{\circ}$ relative to the horizontal axis and nearest neighboring chains are rotated about $90^{\circ}$ relative to each other.

The monoclinic crystal is characterized by three lattice parameters, $a=8.09 \AA, b=4.79 \AA$, and $c=2.53 \AA$ with the tilt angle corresponding to $108^{\circ}$ [22], cf. Fig. 1(b). However, it should be noted that given the notion that the monoclinic lattice is metastable/unstable this data may not correspond to that of zero pressure. The molecular units in the monoclinic unit cell are arranged such that the neighboring chains along the horizontal axis in Fig. 1(b) are rotated by $180^{\circ}$.

To determine the optimal lattice parameters for the two phases, we performed semirelaxed DFT calculations to find the configuration that minimizes the ground state energy. To this end we incrementally varied the lattice parameters and the tilt angle, while allowing all atomic coordinates to relax fully. A conventional relaxation scheme with concurrent stress and atomic coordinate relaxation was not performed due to the fact that the elastic constants vary significantly (by more than one order of magnitude) in the chain and transverse directions, which made it hard to obtain convergence for the stress relaxation.

\section{B. Phase transformation}

In the present work we consider two types of martensitic transformations: $\mathrm{T} 1_{2}$ and $\mathrm{T} 2_{1}[16,22,23]$ (hereafter referred to as $\mathrm{T} 1$ and $\mathrm{T} 2$, respectively). Because the transitions in general 
(a)

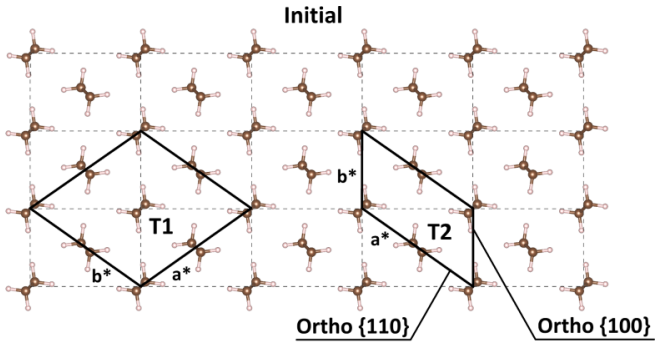

Final

(b)

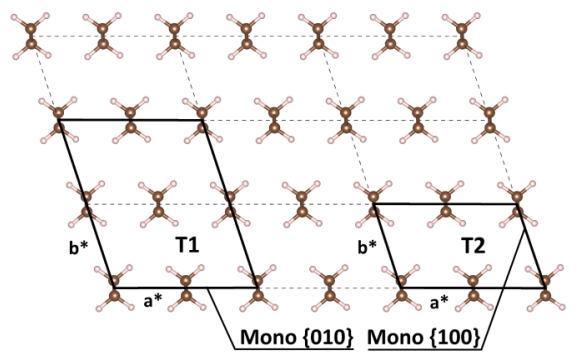

(c)

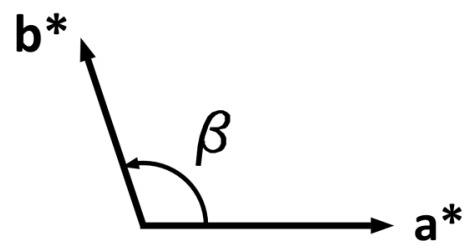

FIG. 1. Illustration of the considered transformation paths from orthorhombic to monoclinic PE viewed along the chain direction. The primitive cells are indicated by the dashed lines. (a) The solid lines indicate the initial supercells for the $\mathrm{T} 1_{2}$ and $\mathrm{T} 2_{1}$ transformation paths and the $\{110\}$ and $\{100\}$ planes of the orthorhombic lattice. (b) Illustration of the final states of monoclinic PE for the transition paths. The solid lines indicate the supercells and the $\{010\}$ and $\{100\}$ planes of the monoclinic lattice. (c) Definition of the supercell vectors $\mathbf{a}^{*}$ and $\mathbf{b}^{*}$ and the tilt angle $\beta$. (a) and (b) Generated using the VESTA code [41].

are nonaffine, the molecular units do not follow the CauchyBorn rule [46], which suggests that the atomic positions cannot be directly mapped along with the deformations. This can be realized for instance by the fact that the nearest neighbor molecular units for the monoclinic phase are rotated by $180^{\circ}$ relative to each other, whereas those of the orthorhombic phase are rotated by about $90^{\circ}$, see Fig. 1 . Hence, we adopted a procedure that enabled us to estimate the strain energy associated with lattice deformations and the chain shuffling activation energy. For the first part we mapped out the ground state energy for different combinations of lattice parameters and tilt angles. Because different molecular chain configurations can minimize the ground state energy for different amounts of strain we tried different initial guesses of the rotational angle around the chain axis for each molecule for each configuration. From this initial state we let the atomic coordinates relax fully while fixing the supercell, such that the atoms were allowed to reach equilibrium for each strain increment. This way we improved the basis for finding the optimal molecular chain configuration for each strain increment, which enabled the identification of the minimum energy lattice deformation path.
Alternative representations of the orthorhombic unit cell are needed depending on the transformation mode considered. Therefore we defined the supercell vectors $\mathbf{a}^{*}$ and $\mathbf{b}^{*}$, and the tilt angle $\beta$ to describe the transverse extension of the supercell, as depicted in Fig. 1(c). For the T1 transformation, we utilized a supercell for the initial state that had twice the volume of the primitive cell, as seen in Fig. 1(a). For this representation the planar supercell vectors $\mathbf{a}^{*}=(a, b, 0)$ and $\mathbf{b}^{*}=(-a, b, 0)$ were used. From the experimental lattice parameters, $\beta$ can be computed to be $112^{\circ}$ for this representation. The other supercell depicted in Fig. 1(a) corresponds to the initial state for the T2 transformation, for which the supercell vectors are $\mathbf{a}^{*}=(-a, b, 0)$ and $\mathbf{b}^{*}=(0, b, 0)$. This implies that the tilt angle between the supercell vectors initially is $124^{\circ}$. For the final state of $\mathrm{T} 1$ we double the volume of the monoclinic primitive cell by letting $b \rightarrow 2 b$, see Fig. 1(b), such that it complies well with the initial state. These representations of the orthorhombic and monoclinic lattices were chosen on the basis that they can be used to model the phase transition, which is reflected by the fact that the tilt angles between the supercell vectors of the initial state are similar to those of the final state.

In principle there are three parameters that need to be varied to fully cover the space of possible transition paths: the lengths of the supercell vectors $\mathbf{a}^{*}$ and $\mathbf{b}^{*}$ and the angle $\beta$ between them. However, to visualize the strain energy landscape we reduce the number of parameters to two by making the third linearly dependent on the others, which requires some elaboration. For T1, because $\beta$ only changes very little (from $112^{\circ}$ for orthorhombic to $108^{\circ}$ for monoclinic) we primarily vary the lengths of the supercell vectors independently to fit those of the orthorhombic and monoclinic phases. To ensure that we concurrently change the tilt angle we vary it linearly with the length of $\mathbf{b}^{*}$. For the T2 transition path, we use an alternative representation to describe the deformation path. Because the length of $\mathbf{b}^{*}$ only changes from about 4.89 to $4.79 \AA$ when transforming from orthorhombic to monoclinic, we instead map the strain energy landscape by varying the length of $\mathbf{a}^{*}$ and $\beta$. To make sure that the lattice parameters of the optimal lattices are met, the length of $\mathbf{b}^{*}$ is varied linearly with $\beta$.

To account for the energy required to perform chain rotations, we used the nudged elastic band (NEB) method $[47,48]$ to compute the activation energy needed for a single chain to rotate in the orthorhombic [see Fig. 1(a)] and in the monoclinic molecular configurations [see Fig. 1(b)]. This approach allowed us to quantify whether the elastic or rotational energies dominate the transformation and enabled us to investigate if the required combined activation energy is low enough for the back transition to be thermally activated.

\section{Computational details}

All DFT simulations in this work were performed using the open-source plane-wave based Quantum Espresso (QE) software suite $[49,50]$. For describing the electron-ion interaction we used the ultrasoft pseudopotential approach $[51,52]$ with the electron description for $\mathrm{C}$ comprising the $2 s^{2} 2 p^{2}$ states, while that of $\mathrm{H}$ consisted of a $1 s^{1}$ electron. We chose the kinetic energy cutoff for the plane-wave basis set and $k$-point density such that the ground state energy was converged to 
within less than $1 \mathrm{meV} / \mathrm{CH}_{2}$ unit. To achieve this, we used a kinetic energy cutoff of $60 \mathrm{Ry}(1 \mathrm{Ry}=13.6 \mathrm{eV})$ and the energy cutoff for the electron density is set to $600 \mathrm{Ry}$. We utilized a $k$-point grid for the first Brillouin zone of the orthorhombic primitive cell corresponding to a $\Gamma$-centered $6 \times 6 \times 12$ sized grid (for the reciprocal $a \times b \times c$ lattice), generated by means of the Monkhorst-Pack method [53]. This was found sufficient to produce well-converged results. The $k$-point mesh for the supercell used for the monoclinic primitive cell was made commensurate with the orthorhombic primitive cell.

For the phonon calculations we adopted the density functional perturbation theory approach as implemented in $\mathrm{QE}$ [54]. To prevent boundary induced artifacts from the periodic conditions, phonons were computed using supercells with twice the volume of the primitive cell, i.e., those depicted to the left in Figs. 1(a) and 1(b) for the orthorhombic and monoclinic phases, respectively. The dynamical matrices and force constants were computed and evaluated on a $2 \times 2 \times 6$ $q$-point grid, from which the phonon dispersion curves were interpolated. To improve the accuracy of the computed force constants, we enforce the acoustic sum rule. The explicit algorithm that is used for this purpose is a subspace projected approach for finding the optimal solution of the coupled acoustic sum rules, as outlined by Mounet [55]. This modeling strategy and employed $q$-point density was found to yield well-converged phonon data.

For investigating the threshold energy associated with chain rotation in the orthorhombic and monoclinic phases, we used the NEB method $[47,48]$ to obtain the activation energy. For the simulations we study the gradual rotation of a single chain from $0^{\circ}$ to $180^{\circ}$ around the axis through the chain's center of mass, as illustrated in Fig. 2. To this end we utilized 11 (one initial, nine intermediate, and one final) images to represent the gradual rotational movements. To avoid artifacts emanating from finite size we considered relatively large supercells with sides not smaller than $14 \AA$, as shown in Fig. 2. Moreover, to prevent unnaturally high repulsion from the neighboring chains that may corrupt the results, we allowed all atoms in the supercell to undergo relaxation. This approach, combined with the chosen supercell sizes, was found to yield an activation energy converged within less than $5 \mathrm{meV} / \mathrm{CH}_{2}$ accuracy, which is sufficient for the present investigation.

The choice of exchange-correlation functional for soft and sparse matter is a delicate issue that requires some elaboration. Since modeling of the martensitic transformation and phonons in PE are typical soft- or sparse-matter problems, we needed to employ a suitable exchange-correlation functional for an accurate description of the interatomic interaction. Owing to the importance of vdW interaction in the considered system, a formulation of the vdW-DF method [37,38,56-60] was considered appropriate.

In the present work we used the recent consistent-exchange vdW-DF-cx formulation [40]. There are formal arguments to expect a high degree of transferability for this nonlocal exchange-correlation formulation, also when materials (such as PE) combine regions with both dense and sparse electron concentrations [39,59]. Also, it has shown high predictive capabilities in terms of reproducing the librational phonon modes of organic crystals [61] and robustness when it comes to providing restoring forces for strained bond lengths [62]. For (a)

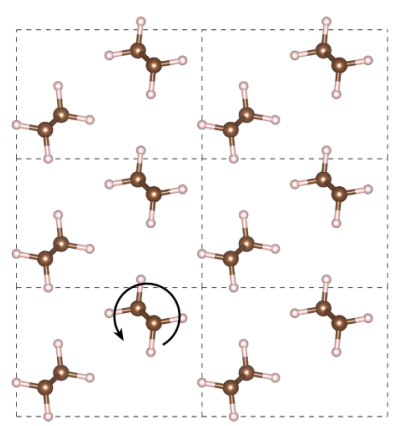

(b)

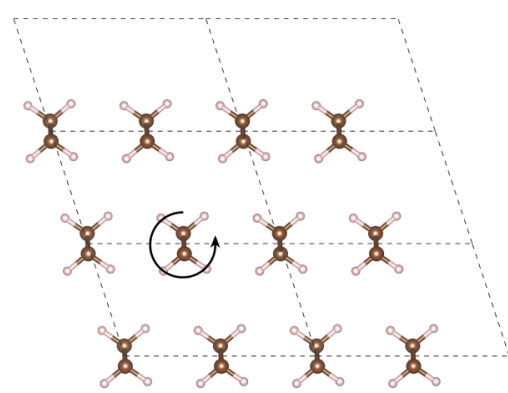

FIG. 2. Schematic illustration of supercell size and the chain shuffling considered for the NEB calculations of the (a) orthorhombic and (b) monoclinic phase.

the case of PE, the vdW-DF-cx functional has shown an ability to predict both the lattice parameters and elastic constants of orthorhombic PE in good agreement with experimental data, where local or semilocal formulations, such as conventional LDA or GGA, fail [21]. Finally, as demonstrated below, in the present work we observed a good agreement between experimental and vdW-DF-cx characterizations of the orthorhombic phonon data. These traits suggest that vdW-DF-cx is a suitable functional choice for the all-theory characterization of the PE martensitic transformation and relative phase stability that we pursued here.

\section{RESULTS}

\section{A. Ground state data}

The results of the lattice optimizations show that the difference in ground state energy (without zero point correction) between the two phases is about $1.5 \mathrm{meV} / \mathrm{CH}_{2}$ unit in favor of the orthorhombic phase. This concurs with the fact that the orthorhombic lattice is most commonly observed. For this phase we have summarized the optimal lattice parameters in Table I together with computed and experimental data from other sources. The obtained results are found to agree quite well with the experimentally measured data. For the initial configuration of the T1 transformation, $\beta$ corresponds to $110^{\circ}$, agreeing well with the experimental data of $112^{\circ}$. Likewise for the initial T2 configuration the tilt angle becomes $125^{\circ}$, which agrees with the experimental data of $124^{\circ}$.

Compared with the vdW-DF-cx results, the general observation is that the LDA data underestimate the transverse 
TABLE I. Computed and experimentally measured lattice parameters and one of the elastic constants $\left(C_{11}\right)$ of the orthorhombic system. The data is presented in $\AA$ and GPa, respectively.

\begin{tabular}{lcccc}
\hline \hline Source & $a$ & $b$ & $c$ & $C_{11}$ \\
\hline DFT (vdW-DF-cx, this work) & 7.19 & 5.02 & 2.55 & 9.0 \\
DFT (LDA [63]) & 6.73 & 4.53 & 2.52 & \\
DFT (LDA [64]) & 6.75 & 4.65 & 2.53 & 16.0 \\
DFT (LDA [65]) & 6.55 & 4.45 & 2.52 & \\
DFT (PBE-GGA, this work) & 8.13 & 5.59 & 2.56 & \\
DFT (PBE-GGA [63]) & 8.28 & 5.64 & 2.57 & \\
DFT (PBE-GGA [64]) & 8.43 & 5.57 & 2.56 & \\
DFT (PBE-GGA [65]) & 7.30 & 5.02 & 2.57 & 39.2 \\
DFT (vdW-DF [66]) & 7.30 & 5.22 & 2.57 & \\
DFT (vdW-DF [67]) & 7.34 & 5.19 & 2.58 & \\
DFT (vdW-DF2 [67]) & 7.05 & 4.98 & 2.58 & \\
DFT (DF-C09 [67]) & 6.84 & 4.76 & 2.55 & \\
DFT (DF-optB88 [67]) & 6.93 & 4.85 & 2.56 & \\
DFT (DF-optB86b [67]) & 6.95 & 4.86 & 2.56 & \\
DFT (revDF2 [67]) & 6.97 & 4.88 & 2.56 & \\
DFT (rVV10 [67]) & 6.90 & 4.77 & 2.56 & \\
Expt. (x-ray, 77 K [44]) & 7.42 & 4.96 & - & \\
Expt. (x-ray, 77 K [42]) & 7.39 & 4.93 & 2.54 & \\
Expt. (neutron, 4 K [43]) & 7.12 & 4.85 & 2.55 & \\
Expt. (neutron, 90 K [43]) & 7.16 & 4.87 & 2.55 & \\
Expt. (neutron, 10 K [45]) & 7.12 & 4.84 & - & \\
Expt. (neutron, 300 K [45]) & 7.42 & 4.94 & - & \\
Expt. (phonons [68]) & & & & 11.5 \\
Expt. (ultrasonic [69]) & & & & 8.4 \\
\hline \hline
\end{tabular}

lattice parameters (i.e., $a$ and $b$ ) by the order of $0.5 \AA$. This deficiency is believed to be due to the well known overbinding and the lacking of weak vdW forces [75,76], which limit the applicability in sparse matter. Depending on the literature source, the reported performance of GGA calculations varies significantly. It has been reported that the lattice parameters are either largely overestimated (cf. [63,64] and this work) or in good agreement with experimental data (cf. [65]), see Table I. However, despite good agreement with experimental lattice parameters, such calculations typically suffer from other deficiencies. In particular the computed elastic constants associated with transverse deformation are substantially overestimated. For instance in [65], the reported value of $C_{11} \approx 40 \mathrm{GPa}$, while the experimental data lie in the range 8.4-11.5 GPa [68,69], implying that the transverse bonding is not accurately accounted for. This elastic constant relates to the stretching of the orthorhombic $a$-lattice parameter, which we in the present work find to be $C_{11} \approx 9.0 \mathrm{GPa}$ in accordance with experimental data.

In addition to the aforementioned data in Table I, we have compared the obtained optimized lattice parameters for PE with those computed by Kleis et al. [66] and Pham et al. [67] for a wide range of nonlocal formulations. Specifically, we have compiled data for the vdW-DF [37], vdW-DF2 [77], DF-C09 [78], DF-optB88 [79], DF-optB86b [80], revDF2 [81,82], and rVV10 [83] functionals. In terms of predicted lattice parameters, those found in the present work for the vdW-DF-cx compare well with those found for the vdW-DF and vdW-DF2 formulations in the literature. The remaining functionals (DF-C09, DF-opt88, DF-opt86b, revDF2, rVV10) predict a slightly underestimated $a$-lattice parameter, whereas $b$ concurs with experimental data.

The computed lattice parameters for the monoclinic lattice correspond to $a=7.90 \AA, b=4.87 \AA$, and $c=2.55 \AA$, which deviate from the experimental data by less than $3 \%$. As comparison we computed the lattice parameters using PBE-GGA, which resulted in $a=9.04 \AA, b=5.50 \AA$, and $c=2.56 \AA$, which substantially overestimate the experimental data. The computed respective tilt angles correspond to $108^{\circ}$ and $112^{\circ}$, of which the former concurs well with experimental data.

\section{B. Phonons}

To investigate the mechanical stability of the two phases, we compute the phonon dispersion and density of states, see Figs. 3 and 4.

\section{Orthorhombic phase}

The phonon branches of the orthorhombic phase can be seen in Fig. 3(a). The fact that no branches assume soft modes for the orthorhombic phase implies that it is stable. Because phonons can be sensitive to the choice of the pseudopotentials and the exchange-correlation functional used to generate them, we validate the predictability by comparing the transverse and longitudinal branches for the path between the $\Gamma$ and $Z$ symmetry points [74] with experimental data [70,71]. The computed results reproduce the experimental data for the acoustic and librational modes with an overall good match, see Fig. 3(b).

Translational modes can be problematic to model with DFT, especially when the intermolecular interaction is weak. The phonon frequencies for the lowest $B_{1 u}$ and $B_{2 u}$ translational modes at the $\Gamma$ point [see Fig. 3(c)] can serve as a test case. Their phonon frequencies are identified in Fig. 3(b) and they correspond to 8.9 and $11.5 \mathrm{meV}$, respectively, which are underestimated by up to about $15 \%$ compared to the respective measurements of 9.9 and $13.4 \mathrm{meV}[72,73]$ at low temperature. However, for higher frequency modes this discrepancy is reduced, as seen for instance by tracing the associated phonon branches in Fig. 3(b). This suggests that the $B_{1 u} / B_{2 u}$ deviation is an upper bound on the errors made in our translational phonon characterization. This is in line with the results reported for crystalline naphtalene in [61], where the discrepancy between experiment and modeling was found to be about $10 \%$ and occurred for the low frequency translational modes.

As a consequence that we consider a cell with twice the volume of the primitive cell, we find that there are nine antiparallel rocking modes and four librational optical modes displaying chain rotational displacements [the latter corresponding to those depicted in Fig. 3(d)]. It should be noted that modes 14 and 15 are merely translated and rotated in the supercell, which means that they are degenerate. The librational modes are important for describing the chain rotations associated with orthorhombic to monoclinic transformation. As can be seen for modeshapes 12 and 14-16, the chain rotations are arranged such that they rotate in the same direction (such as in inset 12 in Fig. 3(d)) or in opposite directions [insets 14-16 in Fig. 3(d)] and they need not display the same displacement amplitude 
(a)

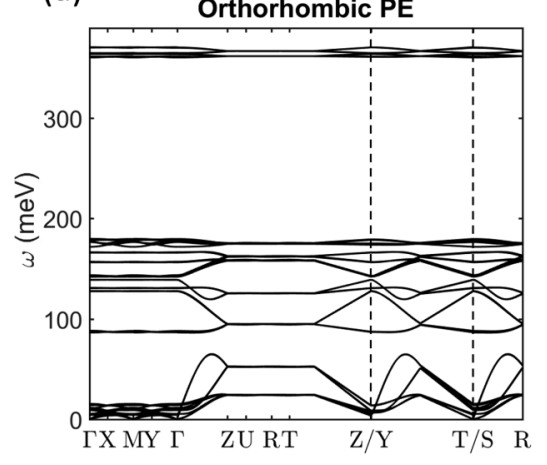

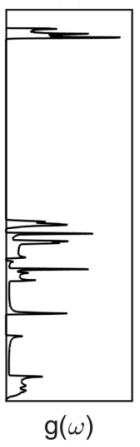

(c)

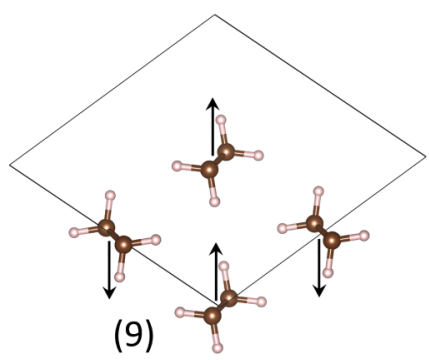

(d)
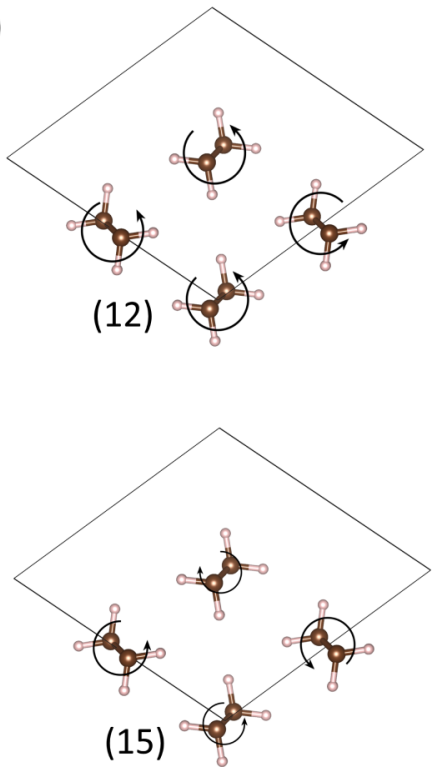

(b)

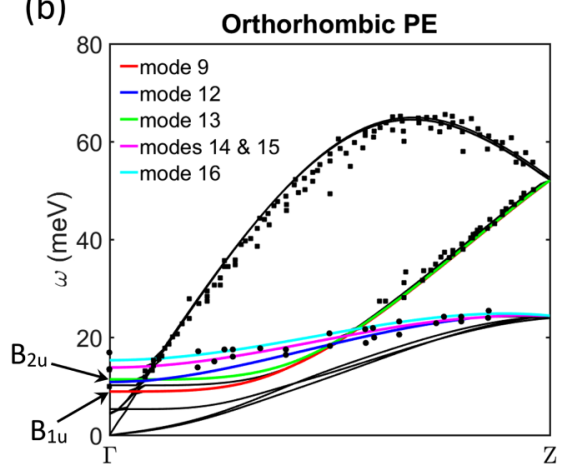

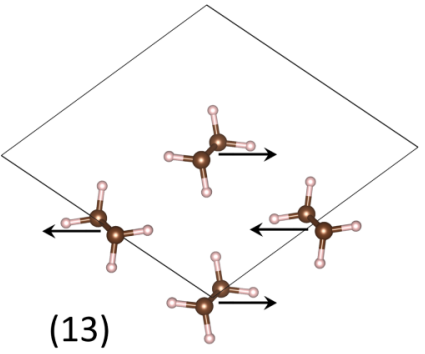

(13)
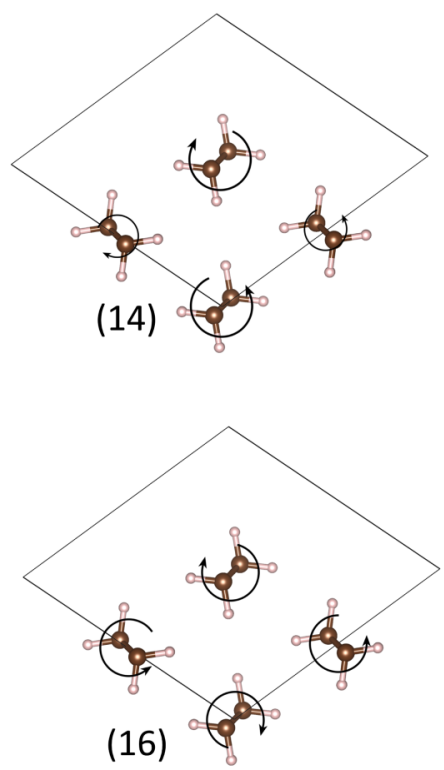

FIG. 3. (a) Phonon dispersion and density of states for stress-free orthorhombic PE along with (b) a high resolution image the low frequency phonon dispersion branches for the $\Gamma-Z$ path compared with experimental results [70-73]. The $k$-vector notation and Brillouin zone paths are adapted from [74] with branches associated with modes 9 and 12-16 as well as the $B_{1 u}$ and $B_{2 u}$ translational intermolecular modes highlighted. (c) Illustration of modes 9 and 13 at the $\Gamma$ point that correspond to the lowest $B_{1 u}$ and $B_{2 u}$ translational intermolecular modes, respectively. The arrows indicate the translational directions of the individual chains. (d) Illustration of the librational intermolecular modes 12 and 14-16 computed at the $\Gamma$ point, which constitute the optical modes with rigid chain rotations around the [001] axis (note that 14 and 15 are degenerate). The arrows indicate the rotational directions of each chain and the size of arrows indicate the relative magnitude.

as the neighboring chain [insets 14 and 15 in Fig. 3(d)]. From Fig. 3(b) it is seen that the branches associated with the librational modes fit the experimental data well. This confirms that the utilized phonon modeling approach has satisfactory predictive capabilities for the current study.

\section{Monoclinic phase}

In light of the conjecture that the monoclinic phase may be a stress induced phase we have studied how the phonon spectrum varies with applied transverse strain. To this end we applied normal strains $\epsilon_{a}$ and $\epsilon_{b}$, such that the lengths of $a$ and 

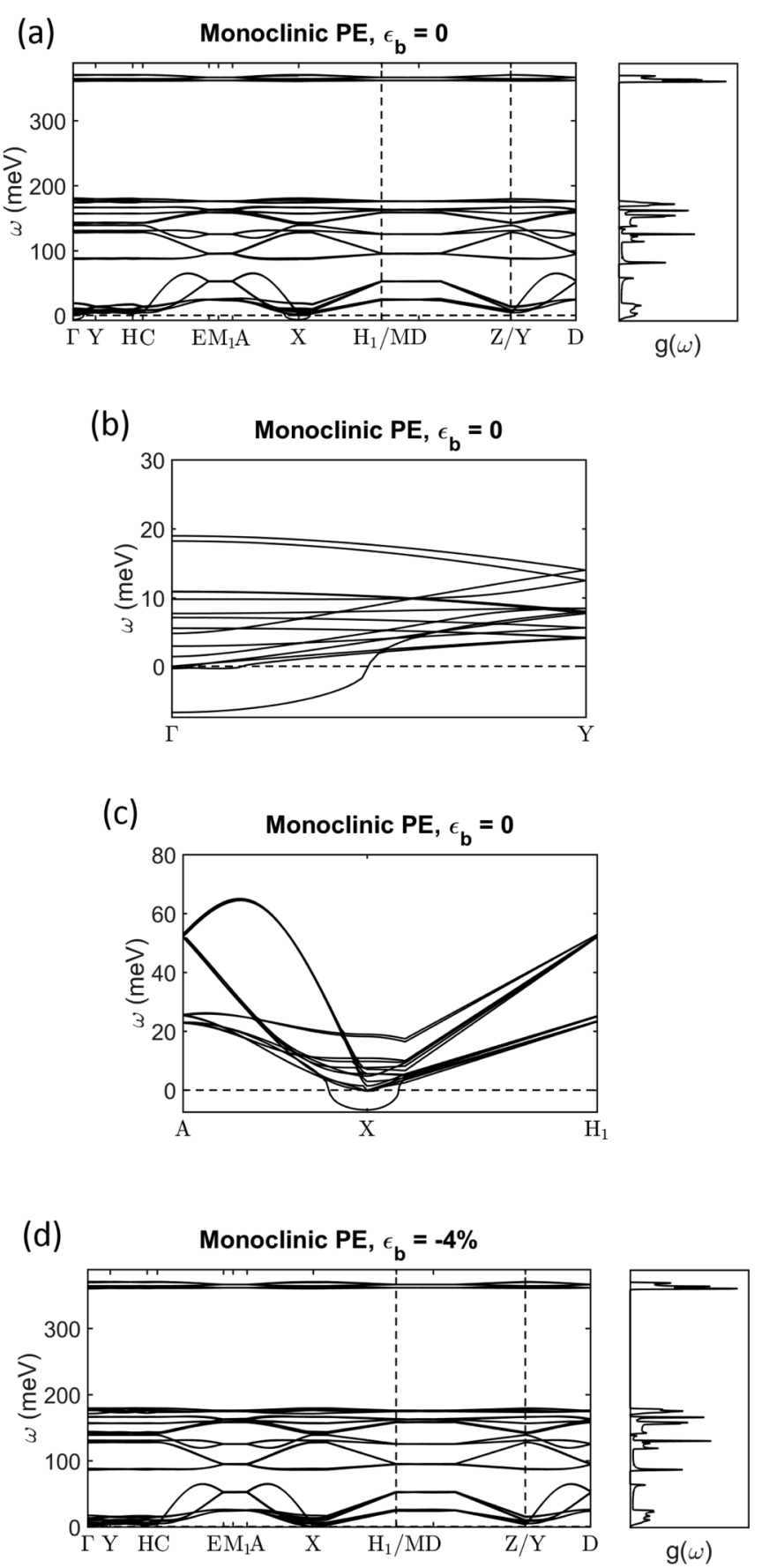

FIG. 4. Phonon dispersion and density of states for monoclinic PE for (a) $\epsilon_{b}=0$. High resolution image of the low frequency phonon dispersion branches in the proximity of (b) $\Gamma$ and (c) $X$ for $\epsilon_{b}$ illustrating the soft mode. The imaginary frequencies are indicated as negative frequencies in (a)-(c). Phonon dispersion and density of states for monoclinic PE for (d) $\epsilon_{b}=-4 \%$.

$b$ were varied. Even though we tested different combinations of such strains, we only present the results for two states corresponding to $\epsilon_{b}=0$ and $\epsilon_{b}=-4 \%$, respectively, see Fig. 4. These states were found to be representative for all considered combinations.

For the stress-free situation there are soft modes present that suggest that the phase is dynamically unstable, see
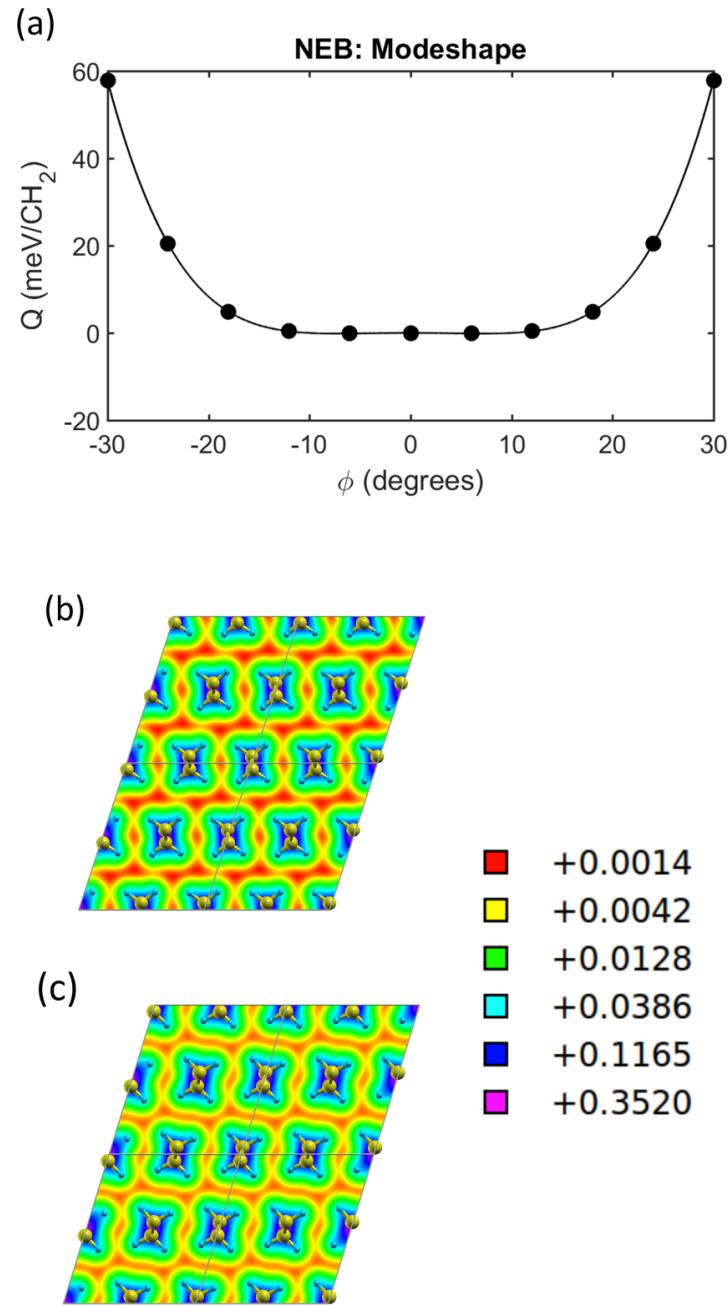

FIG. 5. (a) Activation energy curve $Q$ for concerted modeshape displacements of the soft mode computed by means of NEB. The end points were not allowed to undergo any relaxation, which suggests that the energy levels for $\phi= \pm 30^{\circ}$ are overestimated. Logarithmic charge density plots for (b) $\epsilon_{b}=0$ and (c) $\epsilon_{b}=-4 \%$ with volume specific density levels in units of $e / a_{0}^{3}$, where $e$ denotes the number of electrons and $a_{0}$ is the Bohr radius. The charge density plots are generated using the XCrysDen software [84].

Figs. 4(a)-4(c). Specifically, the imaginary frequencies appear at the $\Gamma$ and $X$ symmetry points, which are situated at the origin and the Brillouin zone boundary, respectively (for details see [74]). To gain insight on the molecular movements associated with the soft mode, we investigate the atomic displacements for the $\Gamma$ and $X$ wave vectors. The modeshape is found to be the same type for both wave vectors: a librational mode represented by chain rotations around the center of gravity, of the same type as depicted in Fig. 3(d) inset 16.

To investigate the physics behind the soft modes, we utilize the NEB method and simulate a concerted shuffling mechanism that reproduces the modeshape of the imaginary mode. To this end we rotate each chain by gradually increasing the rotation angle in the direction indicated by the modeshape. The results from this analysis reveal that the potential energy landscape is virtually flat when the rotation angle is varied between $-15^{\circ}$ to $15^{\circ}$, see Fig. 5(a). This indicates that the chains can 
wiggle back and forth without resistance, which implies that the monoclinic phase is not stable in the low temperature limit. However, it is possible that the phase can be entropically stabilized by phonon-phonon interactions resulting from an increase of the temperature, which would suggest that the phase is metastable at most. Such behavior has been observed for the stabilization of high-temperature body-centered-cubic phases in metals $[85,86]$ and to model this one has to utilize methods that captures anharmonic or even nonadiabatic effects, which lies beyond the scope of this paper.

If we compress the $b$ lattice parameter by $4 \%$, the soft modes disappear, see Fig. 4(d). In effect, the monoclinic phase becomes stable if the lateral dimensions are reduced such that the molecular chains are prevented from rotating. Such transverse deformations can appear as a Poisson effect when the polymer is strained in the chain direction. This result could explain why the phase is only seen when the material is subjected to loading and why it disappears upon relaxation and/or annealing.

To further explore the mechanisms responsible for this behavior, we have studied the link between coordinate changes and charge distributions for both $\epsilon_{b}=0$ and $\epsilon_{b}=-4 \%$, see Figs. 5(b) and 5(c), respectively. The unstrained cell is characterized by regions of depleted charge density, suggesting that the $\mathrm{H}-\mathrm{H}$ repelling is not severe. Our results show that the chains tilt to align with the $b$-lattice vector, when subjected to compressive strain, see Fig. 5(c). Based on a comparison of the charge density plots, we interpret the tilting as occurring because the Pauli principle becomes increasingly more relevant with compression. This is due to a reduced interatomic distance between $\mathrm{H}$ atoms of neighboring molecular chains and a disappearance of the charge-depleted regions arising in concert with the tilting. In this tilted state, the polymer chains are pinned by the surrounding repelling $\mathrm{H}$ atoms of the neighboring molecules. They are then prevented from wiggling freely, which causes the phonon instabilities to disappear.

\section{Orthorhombic-monoclinic transformation}

For the transformation between the two phases to occur, two things need to happen: (i) the lattice needs to deform and (ii) the molecular chains need to shuffle such that the chain arrangements agree with those of the ground state structures. Since both mechanisms contribute to the threshold energy of the transformation we need to quantify them. Below we investigate their separate contributions.

\section{Lattice deformation}

For the lattice deformations it turned out that all lowest energy equilibrium configurations originated from molecular arrangements where the chains were either organized as those of the orthorhombic unit cell [i.e., approximately $90^{\circ}$ rotated, see Fig. 1(a)] or as the monoclinic cell [i.e., aligned with a $180^{\circ}$ skeletal rotation, see Fig. 1(b)]. Thus, in practice the strain energy landscape reflects the competition of lowest ground state energy of different chain arrangements for varied lattice parameters. Therefore to present the strain energy landscape we define the strain energy amplitude associated with each strain increment as $E=\min \left(E_{\text {ortho }}, E_{\text {mono }}\right)$, i.e., we take the lowest ground state energy of the relaxed configurations emanating from initial states with chains arranged according to either the orthorhombic or the monoclinic cell. The reference energy corresponds to that of the ground state of the orthorhombic phase.

Figure 6 shows that for both transition paths, there is a saddle point in the strain energy at which the chain arrangement preference is changed. For both cases this is found to be $\sim 4 \mathrm{meV} / \mathrm{CH}_{2}$ unit. Inspection of the coordinate variation shows that the saddle point serves as a distinct point where the rotated molecular configuration of the orthorhombic phase obtains a higher ground state energy than the aligned molecular configuration of the monoclinic cell, cf. Figs. 6(a) and 6(b). Thus, the saddle region effectively is the border between the two possible molecular chain arrangements and indicates where it is energetically favorable for the molecules to rearrange. This switch is from an arrangement where nearest neighboring chains are rotated by about $90^{\circ}$ to that of the monoclinic cell, where the chains are organized according to Fig. 1(b).

The fact that the isovolume curves in Fig. 6 to a great extent are perpendicular to the energy isolevels and approximately pass through saddle points, indicates that the deformations are approximately volume conserving. This implies that the supercell deformation can be described in terms of a pure shearing mode accompanied by chain rotations, as proposed by Bevis and Crellin [22].

As indicated by the insets in Fig. 6, at the saddle point (i.e., insets a4 and b4 in Fig. 6), the molecular chains are tilted, similar to Fig. 5(c). But as the lattice parameters approach those of the monoclinic (i.e., insets a6 and b6), the molecular chains adapt the orientation of the stress-free monoclinic phase as indicated in Fig. 1(b). Although this tilting can be seen as concerted chain shuffling, it does not reflect the fact that the molecules need to reorient from a nonaligned to an aligned chain configuration, which in the next section is shown to require much higher activation energy.

To better understand the nature of the strain energy landscapes, we study the charge density distributions at minima and saddle points. We find that high ground state energy configurations are accompanied by the formation of regions of depleted charge density as well as those of unusually high charge density. The high density regions are particularly noticeable whenever the separations of $\mathrm{H}$ atoms from neighboring molecules are small, which cause the concerted chain tilting. This can be realized for instance by tracing the charge density plots along the constant volume curves in Fig. 6 from the stable orthorhombic structure to the monoclinic. During this transition, the amount of low and high density regions increases substantially, unless the molecules gradually reorient to reduce the ground state energy.

The fact that there is an emergence of regions of reduced charge density during the transition implies that vdW interaction is the main contributor to the landscape variation. To investigate this closer, Fig. 7 documents the total strain energy $E$ and vdW energy $E_{\mathrm{c}}^{\mathrm{nl}}$ contributions to the optimal isovolume T1 path. The latter of these contributions represents the extent to which the cohesion arises as a partial contribution, originating from the vdW-DF-cx nonlocal correlation energy 
(a)

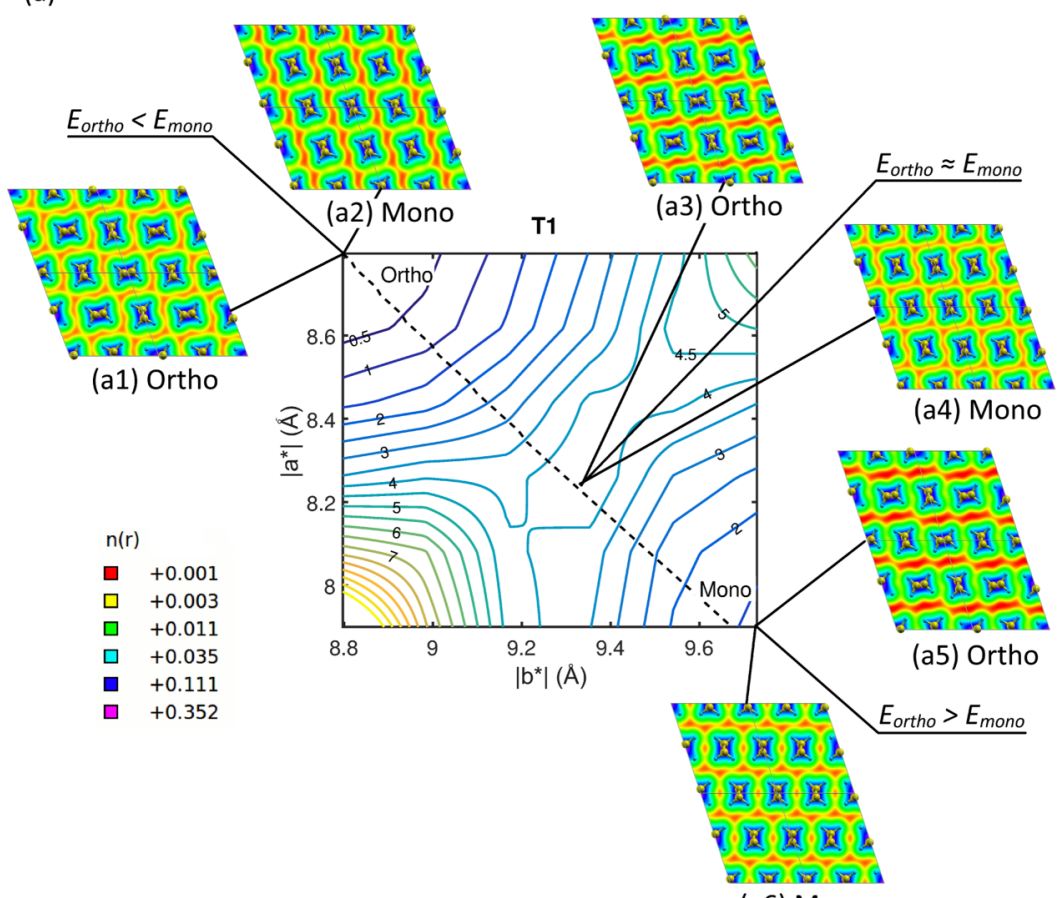

(a6) Mono

(b)

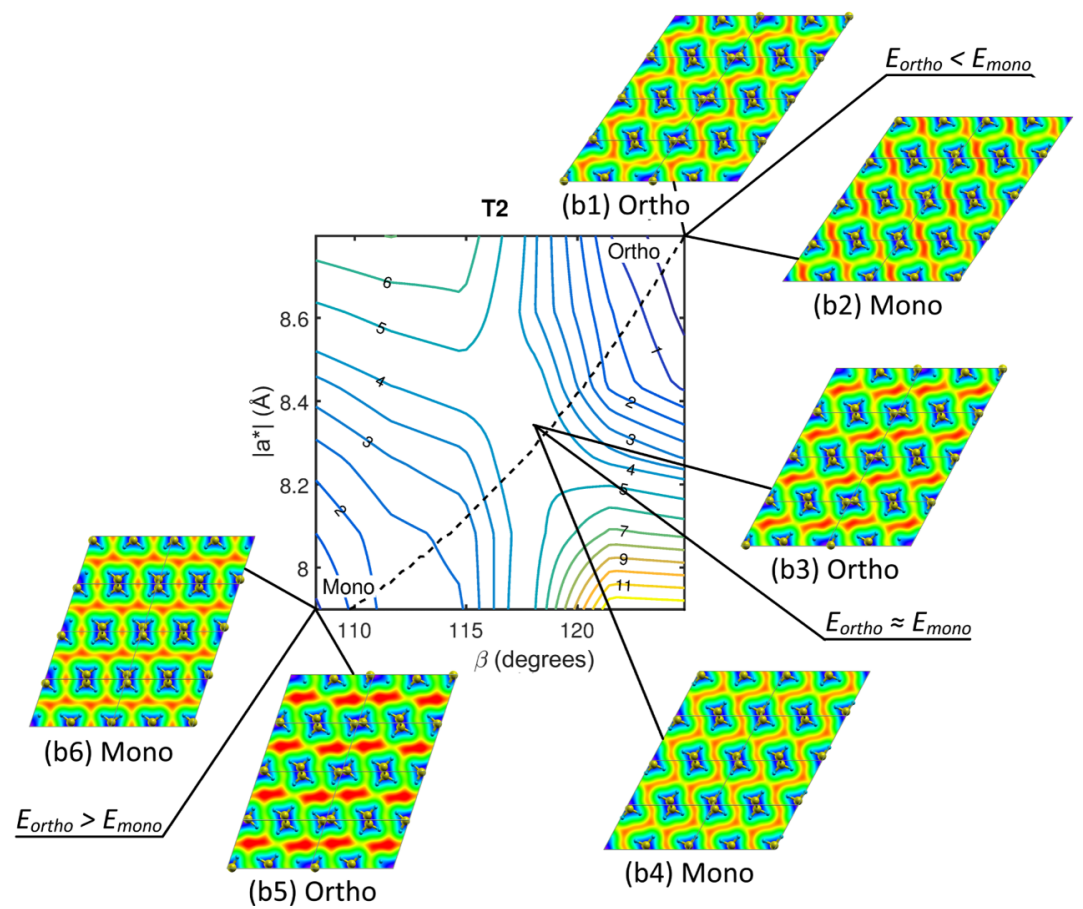

FIG. 6. Strain energy landscapes for (a) T1 and (b) T2 deformation mechanisms. The strain energy $E$ is measured in $\mathrm{meV} / \mathrm{CH}_{2}$ unit, with an uncertainty of less than $\pm 0.5 \mathrm{meV} / \mathrm{CH}_{2}$ unit. The dashed lines correspond to isovolume curves. The insets correspond to logarithmic charge density plots for the relaxed equilibrium configurations using orthorhombic and monoclinic molecular arrangements as initial states. The units for the density levels are the same as in Fig. 5 and the legend applies for both (a) and (b). term. This term describes the vdW interactions in the system and it reflects the interchain vdW binding in the PE crystal. Analysis of Fig. 7 reveals that the peak strain energy and the majority of the overall behavior can be attributed to the $\mathrm{vdW}$ forces, and thus the vdW interaction constitutes the major contribution to the strain energy. The fact that the end points of the curves in Fig. 7 overlap is an indication that the ground state energy difference between the two phases is solely dictated by the vdW interaction.

\section{Chain shuffling}

The second contribution to the threshold energy associated with martensitic transformation is the energy required for the chains to rearrange from a nonaligned configuration to an aligned. To quantify this contribution we consider the activation energy needed for a single chain to rotate $180^{\circ}$. To estimate the activation energy we use the NEB method to compute the energy required for rotating a molecular chain in 


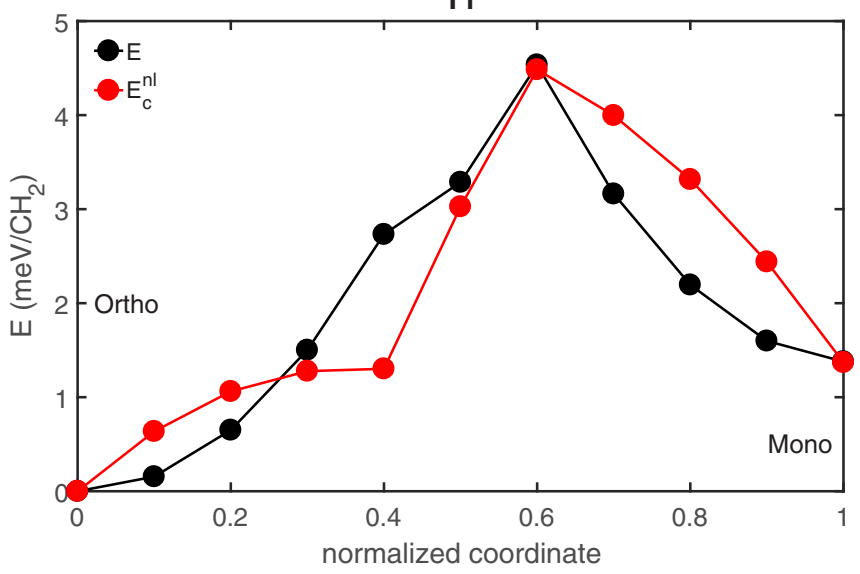

FIG. 7. Strain energy curve of the optimal T1 lattice deformation path and documentation of key role of vdW forces. The set of black markers identify computations of the total strain energy (per polymer repeat unit) in the PE crystal for a set of representative lattices along the isovolume curve of Fig. 6(a). The set of red markers shows the strain energy contributions that arise alone from the nonlocal correlation energy part, i.e., the part of the vdW-DF-cx functional that captures the vdW binding.

the equilibrium orthorhombic and monoclinic phases. Figure 8 shows that the threshold for these transitions lies in the range of $35-50 \mathrm{meV} / \mathrm{CH}_{2}$, which is approximately ten times higher than the threshold energies associated with lattice deformation. Thus, individual chain shuffling dominates and represents the bulk of the threshold energy associated with the martensitic transformation.

The NEB calculations further reveal that the activation energy for rotation is less in the monoclinic configuration, which indicates a preference for rotation in that state. The fact that there are local minima for the rotation corresponding to $90^{\circ}$, implies that chain arrangements with stems aligned or

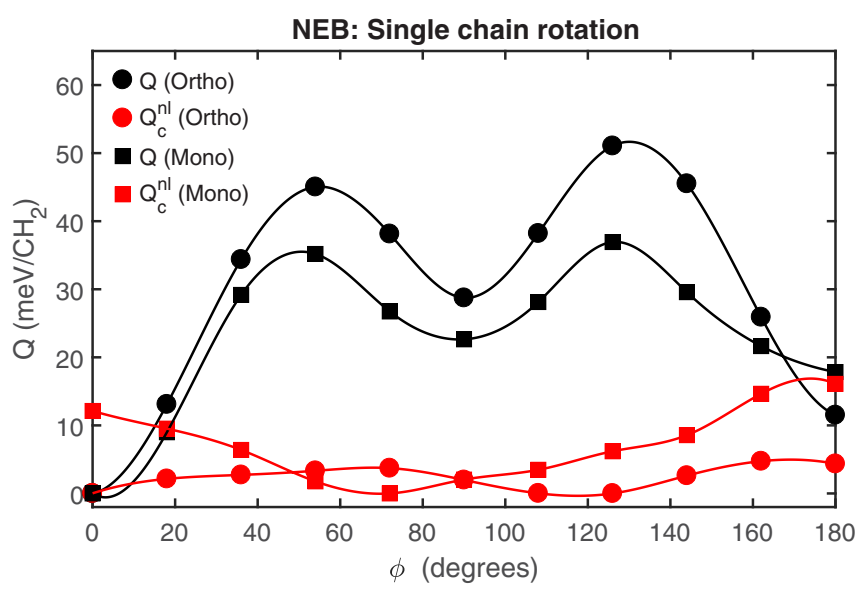

FIG. 8. Estimated activation energies $Q$ for a single chain rotation in the equilibrium orthorhombic and monoclinic phases, computed for the ideal equilibrium lattice parameters of the respective phases by means of the NEB method. The black markers indicate the total energy, whereas the red markers represent the nonlocal correlation contribution. perpendicular are in a (potentially) metastable state. In the same manner as for the lattice deformation, we quantify the $\mathrm{vdW}$ contributions to the activation energy in Fig. 8. It is found that they are relatively small in comparison with the total activation energy, of which they constitute about $10 \%$. Hence, other mechanisms, such as repulsion from close ranged $\mathrm{H}$ atoms of neighboring chains, are more important when describing the chain shuffling activation energy.

\section{DISCUSSION}

In the present section we discuss the main findings and relate them to experimental observations. The results of our nonempirical modeling reveal primarily two very interesting observations: (i) soft phonon modes of the monoclinic phase and (ii) low strain energy required to transform from the orthorhombic to the monoclinic phase accompanied with relatively high activation energy needed for chain shuffling.

The soft phonon modes associated with the monoclinic phase is an indication that it is not stable in the low temperature limit. Based on the analysis of the associated modeshape, the soft modes emanate from the fact that the activation energy for the modeshape movement is zero. This can give rise to a spontaneous wiggling chain motion that manifests in imaginary phonon modes. Although it is beyond the scope of this work to study, it is possible that the phase can be stabilized by phonon-phonon interaction if the temperature is increased.

In characterizing the chain shuffling process (illustrated in Fig. 2) we have chosen to focus on atomic displacements that are similar, although not identical, to those of the phonon mode [labeled 16 in Fig. 3(d)] that is found unstable for the monoclinic phase. Our results for the chain shuffling threshold reflect a Born-Oppenheimer (BO) description that is directly available in ground state DFT, while the phonon analysis is a beyond-BO characterization of stability. The differences between the BO and phonon characterizations of monoclinic phase stability make it desirable to eventually seek a DFT-based account of the nonadiabatic atomic dynamics, see for instance [85-87]. The strong performance for descriptions of the relevant soft-matter librational modes suggests that vdW-DF-cx would be useful, but such an exploration is beyond the present scope. However, as discussed below, many experimental observations can be understood already from the present DFT-based results.

The fact that the soft modes disappear when subjected to transverse deformation is an indication that the monoclinic phase is stabilized when subjected to loading. This is in accordance with results from experiments that have revealed that the monoclinic phase is only observed under such conditions. The strain induced phonon stability is accompanied by chain tilting that appears as a consequence of reduced $\mathrm{H}$-atom distance of neighboring molecules. The tilting emanates from charge density overlap in the close proximity of closely spaced $\mathrm{H}$ atoms of neighboring molecules. Thus, to reduce the energy, the chains are forced to tilt such that the $\mathrm{H}-\mathrm{H}$ separation is increased. Moreover, such rotations reduce the regions of low charge density and lead to more uniform charge density distributions, which in previous investigations have been found to correlate strongly with reducing the ground state energy in $\mathrm{PE}$ [21]. Once in this tilted state, the closely spaced $\mathrm{H}$ atoms pin the chains and prevent them from wiggling freely, which 


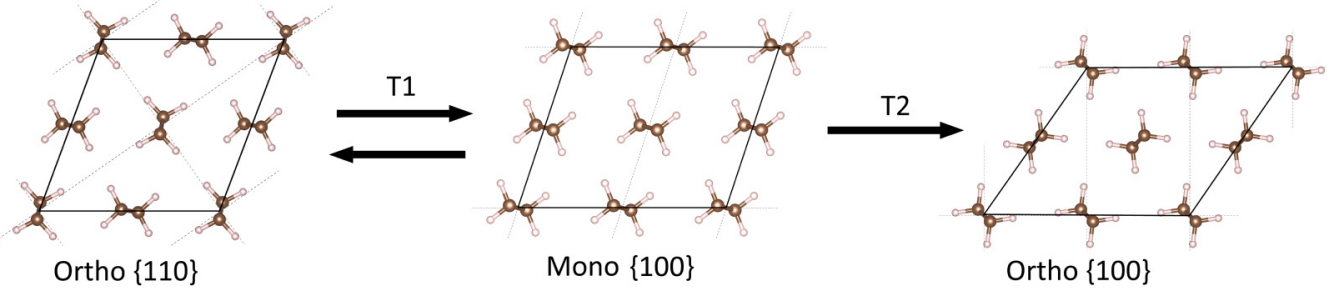

FIG. 9. Possible low threshold energy transition path for lamellae fold planes in crystalline PE. The transition goes from orthorhombic $\{110\}$ to orthorhombic $\{100\}$ via monoclinic $\{100\}$, by the employment of a $\mathrm{T} 1$ transformation followed by a reversed $\mathrm{T} 2$ transformation.

is the reason why the instability modes disappear when the $b$-lattice parameter of the monoclinic phase is subjected to compressive strains. Such strains could emanate either from compressive transverse loads or as a Poisson effect following tensile loading in the chain direction.

The strain energy threshold associated with lattice deformation between the orthorhombic and monoclinic phases is found to be very small, of the order of $4 \mathrm{meV} / \mathrm{CH}_{2}$ unit for both the $\mathrm{T} 1$ and $\mathrm{T} 2$ mechanisms, and it can be exclusively derived from the vdW interaction. These mechanisms do not account for chain shuffling, for which the activation energies were assessed to lie in the range of $35-50 \mathrm{meV} / \mathrm{CH}_{2}$ unit. Thus, the combined energy threshold for both lattice deformation and chain shuffling can be estimated to about $40-55 \mathrm{meV} / \mathrm{CH}_{2}$ unit in the adiabatic description provided by ground state DFT calculations. In order to investigate the possibility of thermally activated back transformation, we compare the threshold energy with that of $k_{B} T$, which is a measure of the averaged kinetic energy. In the temperature range 0 to $300{ }^{\circ} \mathrm{C}$, this translates to about $25-50 \mathrm{meV}$, which is of the same order as the estimated combined threshold energy associated the phase transformation. Hence, it is possible for back transformation to be thermally activated. This could explain the experimental observation of annealing above $110{ }^{\circ} \mathrm{C}$ leading to back transformation from the monoclinic to the orthorhombic phase [35].

The fact that the soft modes of the monoclinic phase can disappear when subjected to relatively small transverse strains could be an indication that it serves as an intermediate configuration that will transform back to the orthorhombic phase when unloaded and relaxed, for instance through the T1 or T2 mechanisms. Since the threshold energy for both mechanisms are of the same order, they may occur with roughly the same probability. This suggests that there are more than one way through which the back transformation from monoclinic to orthorhombic phase can occur following relaxation and/or annealing, as illustrated in Fig. 9. Thus, when loading the orthorhombic phase [such that it transforms to the monoclinic phase (Sec. IIB) via the T1 mechanism], the monoclinic phase can either transform back to the orthorhombic phase via (i) a reversed T1 or (ii) a reversed T2 mechanism upon unloading and relaxation. If (i) occurs the lamellae will have the same fold surfaces as before loading. Such transformation corresponds to that observed in [34], where it was deduced that the interface is of monoclinic(010)/orthorhombic(110) type that moves in the direction of the monoclinic crystal after annealing. However, if (ii) occurs this will lead to a change of the fold-plane type from $\{110\}$ to $\{100\}$, as indicated in Fig. 9. Such reorientation has been observed in crystalline lamellae parts of PE, where the fold planes change from $\{110\}$ to $\{100\}$ following annealing $[7,8,36]$. These observations indicate that both types of transitions between the monoclinic and orthorhombic phase are possible.

\section{SUMMARY AND CONCLUSIONS}

In this work we have investigated the martensitic transformation in crystalline PE by means of first-principles atomistic modeling based on DFT. The modeling is performed using the recent consistent-exchange vdW-DF-cx version $[39,40]$ of the Chalmers-Rutgers vdW-DF method. Besides seeking an accurate characterization of transformation energetics of the martensitic transformation, we have also investigated phonon characteristics of the monoclinic phase to gain insight into its much debated phase stability.

The computed phonon data for the monoclinic phase shows that it is not a completely stable phase. Two instances of imaginary frequencies located at the $\Gamma$ and $X$ symmetry points were observed, with modeshape displacements corresponding to a mode where the chains undergo rotational movements. However, if one of the transverse lattice parameters $(b)$ is subjected to compressive strains, the reduced $\mathrm{H}-\mathrm{H}$ distance between neighboring molecular chains causes regions of increased electron density that through the Pauli principle give rise to chain tilting. In this tilted state the molecular chains are pinned by the repulsive forces emanating from neighboring chains, causing the soft modes to disappear. This concurs with experimental observations of the monoclinic phase being exclusively observed in mechanically loaded specimens and the occurrence of back transformation from the monoclinic to the orthorhombic phase upon relaxation or annealing.

For the martensitic transformation, we have studied the strain energy landscapes for two experimentally observed transformation paths and the activation energy for chain shuffling. The results reveal that the combined threshold energy for these mechanisms are of the same order as $k_{B} T$ (in the range from room temperature to $300{ }^{\circ} \mathrm{C}$ ), which implies that relaxation and thermally activated back transformation between the two phases may occur, as experimentally observed in the literature. Through combinations and reversals of the T1 and T2 mechanisms, we have proposed a transformation path that could explain the experimentally observed reorientation of lamellae fold planes from $\{110\}$ to $\{100\}$ type upon annealing. Moreover, the low activation energy of the lattice deformation and chain shuffling mechanisms are in accordance with the experimentally observed back transformation mechanism that corresponds to the gradual movement of a monoclinic(010)/orthorhombic(110) interface towards the 
monoclinic part of the crystal until it is fully transformed to orthorhombic.

Overall, the martensitic transformation and phase stability of PE is found to reflect a subtle balance of forces $[39,76]$. We have documented that strain energy barriers for the transformation and the difference in ground state energy between the two phases are very low (a few meVs per polymer repeat unit). These energy characteristics can be explained by interchain vdW forces, which dictate the phase stability and strain energy. At the same time, we have shown that a robust account of Pauli exclusion is important to describe the instability and stability of phonon modes and the chain shuffling activation energy that reflect the nature of the monoclinic-to-orthorhombic transformation. Finally, in the stabilization of the monoclinic phase (that arises with compressive strain) the PE chains reorganize themselves to both maximize the vdW attraction and the kinetic-energy repulsion that underpins the Pauli exclusion. These findings demonstrate the importance of the inclusion of $\mathrm{vdW}$ interaction in the present application.

\section{ACKNOWLEDGMENTS}

This work was funded by the Swedish Knowledge Foundation (KKS) through Grant No. 20150165, by the Swedish Research Council (VR) through Grants No. 2014-4310 and No. 2014-5289, and by the Swedish Foundation for Strategic Research (SSF) under Contract No. SE13-0061. The simulations were performed using computational resources provided by the Swedish National Infrastructure for Computing (SNIC) at the National Supercomputer Centre (NSC), Linköping University and at the High Performance Computing Center North (HPC2N), Umeå University.
[1] R. Young and P. Lovell, Introduction to Polymers, Third Edition (Taylor \& Francis, London, 2011).

[2] A. S. Argon, The Physics of Deformation and Fracture of Polymers (Cambridge University Press, Cambridge, 2013).

[3] A. Rozanski and A. Galeski, Int. J. Plast. 41, 14 (2013).

[4] M. Uchida and N. Tada, Int. J. Plast. 49, 164 (2013).

[5] D. R. Askeland, The Science and Engineering of Materials, SI Edition, 3rd ed. (Stanley-Thornes, Cheltenham, 1998).

[6] S. S. Katti and M. Schultz, Polym. Eng. Sci. 22, 1001 (1982).

[7] P. B. Bowden and R. J. Young, J. Mater. Sci. 9, 2034 (1974).

[8] L. Lin and A. S. Argon, J. Mater. Sci. 29, 294 (1994).

[9] Z. Bartczak and A. Galeski, Macromol. Symp. 294, 67 (2010).

[10] J. Petermann and H. Gleiter, Philos. Mag. 25, 813 (1972).

[11] H. Gleiter and A. S. Argon, Philos. Mag. 24, 71 (1971).

[12] A. Cowking and J. G. Rider, J. Mater. Sci. 4, 1051 (1969).

[13] F. C. Frank, A. Keller, A. O'Connor, and H. H. Wills, Philos. Mag. 3, 64 (1958).

[14] V. F. Holland, J. Appl. Phys. 35, 3235 (1964).

[15] R. J. Young, P. B. Bowden, J. M. Ritchie, and J. G. Rider, J. Mater. Sci. 8, 23 (1973).

[16] R. J. Young and P. B. Bowden, Philos. Mag. 29, 1061 (1974).

[17] A. Galeski, Prog. Polym. Sci. 28, 1643 (2003).

[18] A. Galeski, Z. Bartczak, A. S. Argon, and R. E. Cohen, Macromolecules 25, 5705 (1992).

[19] P. Allan and M. Bevis, Philos. Mag. A 41, 555 (1980).

[20] N. A. Geary and D. J. Bacon, J. Mater. Sci. 18, 864 (1983).

[21] P. A. T. Olsson, E. Schröder, P. Hyldgaard, M. Kroon, E. Andreasson, and E. Bergvall, Polymer 121, 234 (2017).

[22] M. Bevis and E. Crellin, Polymer 12, 666 (1971).

[23] P. Allan, E. B. Crellin, and M. Bevis, Philos. Mag. 27, 127 (1973).

[24] P. Allan and M. Bevis, Philos. Mag. 35, 405 (1977).

[25] P. Allan and M. Bevis, Philos. Mag. 31, 1001 (1975).

[26] T. Seto, T. Hara, and K. Tanaka, Jpn. J. Appl. Phys. 7, 31 (1968).

[27] I. L. Hay and A. Keller, J. Polym. Sci. C 30, 289 (1970).

[28] E. R. Walter and F. P. Reding, J. Polym. Sci. 21, 557 (1956).

[29] P. W. Teare and D. R. Holmes, J. Polym. Sci. 24, 496 (1957).

[30] B. Gurun, D. G. Bucknall, Y. S. Thio, C. C. Teoh, and E. HarkinJones, J. Polym. Sci. B 49, 669 (2011).

[31] S. J. Spells and S. Coutry, Appl. Spectrosc. 61, 276 (2007).
[32] H. Kiho, A. Peterlin, and P. H. Geil, J. Appl. Phys. 35, 1599 (1964).

[33] H. Kiho, A. Peterlin, and P. H. Geil, J. Polym. Sci. B 3, 157 (1965).

[34] Y. Takahashi, T. Ishida, and M. Furusaka, J. Polym. Sci. B 26, 2267 (1988).

[35] H. Kiho, A. Peterlin, and P. H. Geil, J. Polym. Sci. B 3, 263 (1965).

[36] Y. Kikuchi and S. Krimm, J. Macromol. Sci.-Phys. B 4, 461 (1970).

[37] M. Dion, H. Rydberg, E. Schröder, D. C. Langreth, and B. I. Lundqvist, Phys. Rev. Lett. 92, 246401 (2004).

[38] K. Berland, V. R. Cooper, K. Lee, E. Schröder, T. Thonhauser, P. Hyldgaard, and B. I. Lundqvist, Rep. Prog. Phys. 78, 066501 (2015).

[39] K. Berland, C. A. Arter, V. R. Cooper, K. Lee, B. I. Lundqvist, E. Schröder, T. Thonhauser, and P. Hyldgaard, J. Chem. Phys. 140, 18A539 (2014).

[40] K. Berland and P. Hyldgaard, Phys. Rev. B 89, 035412 (2014).

[41] K. Momma and F. Izumi, J. Appl. Crystal. 44, 1272 (2011).

[42] S. Kavesh and J. M. Schultz, J. Polym. Sci. A-2: Polym. Phys. 8, 243 (1970)

[43] G. Avitabile, R. Napolitano, B. Pirozzi, K. D. Rouse, M. W. Thomas, and B. T. M. Willis, J Polym. Sci.: Polym. Lett. Ed. 13, 351 (1975).

[44] P. W. Teare, Acta Crystal. 12, 294 (1959).

[45] Y. Takahashi, Macromolecules 31, 3868 (1998).

[46] J. Ericksen, Math. Mech. Solids 13, 199 (2008).

[47] H. Jonsson, G. Mills, and K. W. Jacobsen, Nudged Elastic Band Method for Finding Minimum Energy Paths of Transitions in Classical and Quantum Dynamics in Condensed Phase Simulations (World Scientific, Singapore, 1998), pp. 385-404.

[48] D. Sheppard, R. Terrell, and G. Henkelman, J. Chem. Phys. 128, 134106 (2008).

[49] P. Giannozzi, S. Baroni, N. Bonini, M. Calandra, R. Car, C. Cavazzoni, D. Ceresoli, G. L. Chiarotti, M. Cococcioni, I. Dabo et al., J. Phys.: Condens. Matter 21, 395502 (2009).

[50] P. Giannozzi, O. Andreussi, T. Brumme, O. Bunau, M. B. Nardelli, M. Calandra, R. Car, C. Cavazzoni, D. Ceresoli, M. Cococcioni et al., J. Phys.: Condens. Matter 29, 465901 (2017). 
[51] D. Vanderbilt, Phys. Rev. B 41, 7892 (1990).

[52] K. F. Garrity, J. W. Bennett, K. M. Rabe, and D. Vanderbilt, Comput. Mater. Sci. 81, 446 (2014).

[53] H. J. Monkhorst and J. D. Pack, Phys. Rev. B 13, 5188 (1976).

[54] S. Baroni, P. Giannozzi, and E. Isaev, Rev. Mineral. Geochem. 71, 39 (2010).

[55] N. Mounet, Master thesis, MIT, 2005.

[56] H. Rydberg, M. Dion, N. Jacobson, E. Schröder, P. Hyldgaard, S. I. Simak, D. C. Langreth, and B. I. Lundqvist, Phys. Rev. Lett. 91, 126402 (2003).

[57] M. Dion, H. Rydberg, E. Schröder, D. C. Langreth, and B. I. Lundqvist, Phys. Rev. Lett. 95, 109902(E) (2005).

[58] T. Thonhauser, V. R. Cooper, S. Li, A. Puzder, P. Hyldgaard, and D. C. Langreth, Phys. Rev. B 76, 125112 (2007).

[59] P. Hyldgaard, K. Berland, and E. Schröder, Phys. Rev. B 90, 075148 (2014).

[60] T. Thonhauser, S. Zuluaga, C. A. Arter, K. Berland, E. Schröder, and P. Hyldgaard, Phys. Rev. Lett. 115, 136402 (2015).

[61] F. Brown-Altvater, T. Rangel, and J. B. Neaton, Phys. Rev. B 93, 195206 (2016).

[62] P. Erhart, P. Hyldgaard, and D. Lindroth, Chem. Mater. 27, 5511 (2015).

[63] B. Montanari, P. Ballone, and R. O. Jones, J. Chem. Phys. 108, 6947 (1998).

[64] G. D. Barrera, S. F. Parker, A. J. Ramirez-Cuesta, and P. C. H. Mitchell, Macromolecules 39, 2683 (2006).

[65] M. S. Miao, M.-L. Zhang, V. E. V. Doren, C. V. Alsenoy, and J. L. Martins, J. Chem. Phys. 115, 11317 (2001).

[66] J. Kleis, B. I. Lundqvist, D. C. Langreth, and E. Schröder, Phys. Rev. B 76, 100201 (2007).

[67] T. H. Pham, R. Ramprasad, and H.-V. Nguyen, J. Chem. Phys. 144, 214905 (2016)
[68] J. F. Twisleton, J. W. White, and P. A. Reynolds, Polymer 23, 578 (1982).

[69] C. L. Choy and W. P. Leung, J. Polym. Sci.: Polym. Phys. Ed. 23, 1759 (1985).

[70] D. A. Braden, S. F. Parker, J. Tomkinson, and B. S. Hudson, J. Chem. Phys. 111, 429 (1999).

[71] J. Tomkinson, S. F. Parker, D. A. Braden, and B. S. Hudson, Phys. Chem. Chem. Phys. 4, 716 (2002).

[72] Y. Takahashi, Macromolecules 34, 7836 (2001).

[73] Y. Takahashi, Macromolecules 38, 5356 (2005).

[74] W. Setyawan and S. Curtarolo, Comput. Mater. Sci. 49, 299 (2010).

[75] A. van de Walle and G. Ceder, Phys. Rev. B 59, 14992 (1999).

[76] K. Berland and P. Hyldgaard, Phys. Rev. B 87, 205421 (2013).

[77] K. Lee, E. D. Murray, L. Kong, B. I. Lundqvist, and D. C. Langreth, Phys. Rev. B 82, 081101 (2010).

[78] V. R. Cooper, Phys. Rev. B 81, 161104 (2010).

[79] A. D. Becke, Phys. Rev. A 38, 3098 (1988).

[80] A. D. Becke, J. Chem. Phys. 85, 7184 (1986).

[81] I. Hamada, Phys. Rev. B 89, 121103 (2014).

[82] I. Hamada, Phys. Rev. B 91, 119902(E) (2015).

[83] O. A. Vydrov and T. Van Voorhis, J. Chem. Phys. 133, 244103 (2010).

[84] A. Kokalj, Comput. Mater. Sci. 28, 155 (2003).

[85] P. Souvatzis, O. Eriksson, M. I. Katsnelson, and S. P. Rudin, Phys. Rev. Lett. 100, 095901 (2008).

[86] P. Souvatzis, T. Björkman, O. Eriksson, P. Andersson, M. I. Katsnelson, and S. P. Rudin, J. Phys.: Condens. Matter 21, 175402 (2009).

[87] F. Agostini, S. K. Min, A. Abedi, and E. K. U. Gross, J. Chem. Theory Comput. 12, 2127 (2016). 